\title{
Synthesis of Imine Congeners of Resveratrol and Evaluation of Their Anti-Platelet Activity
}

\author{
Mohammad Bigdeli ${ }^{1}$, Maryam Sabbaghan ${ }^{2}$, Marjan Esfahanizadeh ${ }^{3}$, Farzad Kobarfard ${ }^{3,4, *}$, \\ Sara Vitalini ${ }^{5, *(\mathbb{D})}$, Marcello Iriti ${ }^{5, *(D)}$ and Javad Sharifi-Rad ${ }^{6, *}$ (D) \\ 1 Department of Pharmaceutical Chemistry, Faculty of Pharmaceutical Chemistry, \\ Islamic Azad University-Pharmaceutical Sciences Branch (IAUPS), Tehran 11369, Iran; \\ mo.bigdeli65@gmail.com \\ 2 Chemistry Department, Faculty of Sciences, Shahid Rajaee Teacher Training University, Lavizan 16785-163, \\ Tehran, Iran; sabbaghan@srttu.edu \\ 3 Phytochemistry Research Center, Shahid Beheshti University of Medical Sciences, Tehran 14155-6153, Iran; \\ marjanesfahanizadeh@gmail.com \\ 4 Department of Medicinal Chemistry, School of Pharmacy, Shahid Beheshti University of Medical Sciences, \\ Tehran 11369, Iran \\ 5 Department of Agricultural and Environmental Sciences, Milan State University Via G. Celoria 2, \\ 20133 Milan, Italy \\ 6 Zabol Medicinal Plants Research Center, Zabol University of Medical Sciences, Zabol 61615585, Iran \\ * Correspondence: farzadkf@yahoo.com (F.K.); sara.vitalini@unimi.it (S.V.); marcello.iriti@unimi.it (M.I.); \\ javad.sharifirad@gmail.com (J.S.-R.); Tel.: +98-218-820-0092 (F.K.); +39-2-5031-6766 (S.V.); \\ +39-2-5031-6766 (M.I.); +98-21-88200104 (J.S.-R.)
}

Received: 27 November 2018; Accepted: 20 December 2018; Published: 22 December 2018

\begin{abstract}
Resveratrol (3,5,4'-trihydroxy-trans-stilbene) is a cardioprotective phytochemical occurring in many plant products. In this study, a new series of imine congeners of resveratrol has been synthesized in which the imine moiety replaced the double bond in the structure of resveratrol. In addition, the in vitro antiplatelet activity of these resveratrol derivatives has been evaluated against adenosine diphosphate (ADP), arachidonic acid (AA), and collagen as platelet aggregation inducers. In general, the synthesized compounds were active as antiplatelet agents, and, therefore, the imine functional group may be considered as an effective replacement for a double bond in resveratrol for developing new and promising antiplatelet drugs.
\end{abstract}

Keywords: stilbenes; cardioprotection; antiplatelet drugs; polyphenols

\section{Introduction}

Cardiovascular diseases (CVDs) are recognized as the first global cause of death. It has been reported by the World Health Organization (WHO) that 17.7 million people died from CVDs in 2015 [1,2]. Platelet aggregation plays an essential role in the process of blood clotting and CVDs. However, many antiplatelet drugs such as aspirin and clopidogrel, which are available in clinics, are associated with some side effects such as bleeding and drug resistance that limit their usage $[3,4]$. Therefore, the search for new antiplatelet agents with fewer side effects and higher efficacy is among the priorities of medicinal chemists.

Natural products with various chemical structures have an important role in drug discovery and development [5-9]. Although chemical diversity of the natural products is pivotal in finding useful lead compounds, usually chemical modifications are needed to improve their potency and physicochemical properties [10-12]. Resveratrol (3,5,4'-trihydroxy-trans-stilbene) (1) (Figure 1) is one of these lead compounds. This stilbene is found in many natural sources such as grapes, 
apples, and berries [13-15]. Various biological activities of resveratrol have been reported such as anticancer, anti-inflammatory, antioxidant, and antiplatelet $[13,16,17]$. Orsini et al. synthesized and evaluated the antiplatelet aggregation activity of resveratrol 3-O- $\beta$-D-glucopyranoside and related hyroxystilbenes [18]. Dutra et al. synthesized new resveratrol and resveratrol-furoxan hybrids as antiplatelet and antithrombotic agents [19].

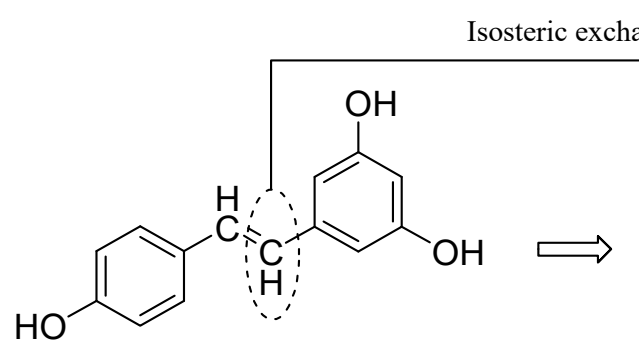

Resveratrol 1

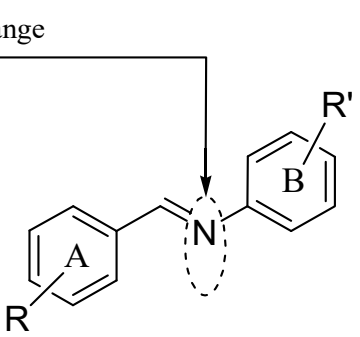

Design Compounds

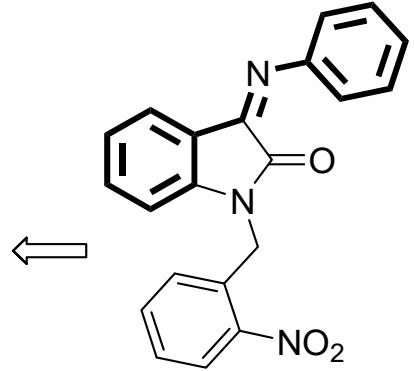

2

Figure 1. Design of imine resveratrol derivatives.

A literature review revealed that $\mathrm{C}=\mathrm{N}$ moiety is present in many structures with antiplatelet activity. Tehrani et al. synthesized a series of Schiff bases derived from 2-hydrazinyl-1,3,4-thiadiazole with high antiplatelet activity [20]. Akhlaghi et al. reported 3-(arylimino)indolin-2-one and 1-(aryl)-3-(phenylimino)indolin-2-one derivatives as antiplatelet agents [21]. Among their synthesized derivatives, compound (2) exhibited high antiplatelet activity against arachidonic acid (AA) as a platelet aggregation inducer $\left(\mathrm{IC}_{50}=3.4 \mu \mathrm{M}\right)$ [21]. Furthermore, antiplatelet activity of $N^{\prime}$-benzylidene-carbohydrazide- $1 H$-pyrazolo[3,4- $b$ ]pyridine derivatives have been reported [22]. A variety of indole hydrazone derivatives such as indole $N$-acylhydrazones [23,24], indole-3-carboxaldehyde phenylhydrazones [25], $N-1$ substituted indolehydrazones [26], indole-3-carbaldehyde, and indole-2-carbaldehayde hydrazones [27] have been previously synthesized in our research group. Some of these reported derivatives exhibited remarkable antiplatelet activity.

Therefore, the present research was aimed at the synthesis of a new series of imine congeners of resveratrol in which the imine moiety replaced the double bond in the structure of resveratrol (Figure 1) [28], and the evaluation of their in vitro antiplatelet activity against adenosine diphosphate (ADP), arachidonic acid (AA), and collagen as platelet aggregation inducers.

\section{Results and Discussion}

\subsection{Chemistry}

The designed compounds were synthesized by the reaction of different aniline derivatives with appropriate aldehydes in water as a green solvent without any catalyst (Figure 2). The synthesized derivatives $(3 \mathbf{a}-3 \mathbf{r})$ were obtained with high yields $(>89 \%)$. Structure of the synthesized compounds was characterized by LC-MS ${ }^{1} \mathrm{H}-\mathrm{NMR}$ and ${ }^{13} \mathrm{C}-\mathrm{NMR}$. The ${ }^{1} \mathrm{H}-\mathrm{NMR}$ spectra of the synthesized compounds exhibited a singlet peak for the $\mathrm{CH}=\mathrm{N}$ proton between 8.51 and $8.96 \mathrm{ppm}$. 


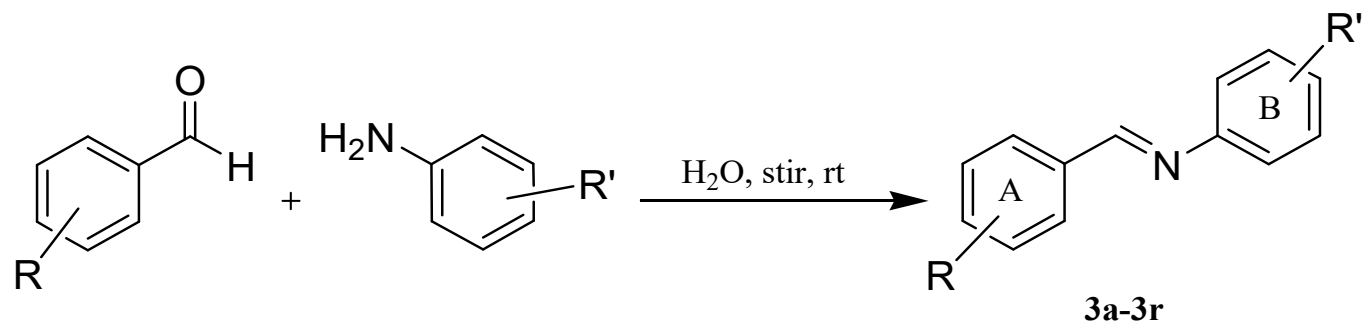

Figure 2. Synthesis of resveratrol derivatives.

\subsection{Anti-Platelet Activity}

The anti-platelet activity of the synthesized derivatives against ADP, AA, and collagen as platelet aggregation inducers were evaluated, according to the Born method $[29,30]$. The obtained data are presented in Table 1.

Table 1. Anti-platelet activity of the synthesized derivatives. Adenosine diphosphate (ADP), arachidonic acid (AA), and collagen were used as a platelet aggregation inducer at a final concentration of $5 \mu \mathrm{M}, 1.35 \mu \mathrm{M}$, and $2.5 \mu \mathrm{g} \cdot \mathrm{mL}^{-1}$, respectively. The results are expressed as the mean \pm standard error of mean (SEM) from three independent experiments.

\begin{tabular}{|c|c|c|c|c|c|c|}
\hline Compound & $\mathbf{R}$ & $\mathbf{R}^{\prime}$ & $\begin{array}{c}\mathrm{AA}^{\mathrm{a}} \\
\text { Inhibition (\%) }\end{array}$ & $\begin{array}{c}\text { AA } \\
\left(\mathrm{IC}_{50} \mu \mathrm{M}\right)\end{array}$ & $\begin{array}{c}\text { ADP }^{\mathrm{a}} \\
\text { Inhibition (\%) }\end{array}$ & $\begin{array}{c}\text { Collagen a } \\
\text { Inhibition (\%) }\end{array}$ \\
\hline $3 a$ & $4-\mathrm{OCH}_{3}$ & - & $74.7 \pm 2.5$ & $301.0 \pm 8.7$ & $43.2 \pm 3.3$ & $15.9 \pm 1.5$ \\
\hline $3 b$ & $3-\mathrm{OCH}_{3}, 4-\mathrm{OH}$ & - & 100 & $180.2 \pm 5.4$ & $52.1 \pm 2.2$ & $39.0 \pm 8.8$ \\
\hline $3 c$ & $2-\mathrm{OH}$ & $4^{\prime}-\mathrm{OCH}_{3}$ & 100 & $86.1 \pm 6.6$ & $30.2 \pm 5.3$ & $46.8 \pm 4.3$ \\
\hline $3 d$ & $4-\mathrm{OCH}_{3}$ & $4^{\prime}-\mathrm{OCH}_{3}$ & 100 & $155.5 \pm 4.3$ & $41.4 \pm 6.4$ & $29.1 \pm 1.9$ \\
\hline $3 e$ & 3,4-dimethoxy & $4^{\prime}-\mathrm{OCH}_{3}$ & 100 & $73.6 \pm 3.4$ & $63.2 \pm 3.1$ & $80.7 \pm 1.4$ \\
\hline $3 \mathrm{f}$ & $4-\mathrm{CH}_{3}$ & $4^{\prime}-\mathrm{OCH}_{3}$ & 100 & $143.3 \pm 6.2$ & $33.6 \pm 1.4$ & $60.0 \pm 4.1$ \\
\hline $3 g$ & $3-\mathrm{OCH}_{3}, 4-\mathrm{OH}$ & $4^{\prime}-\mathrm{OCH}_{3}$ & $94.9 \pm 0.7$ & $179.2 \pm 7.1$ & $34.4 \pm 5.6$ & $41.0 \pm 6.6$ \\
\hline $3 \mathrm{~h}$ & $3-\mathrm{OCH}_{3}$ & $4^{\prime}-\mathrm{OH}$ & 100 & $69.1 \pm 5.4$ & $77.4 \pm 1.9$ & $73.1 \pm 2.1$ \\
\hline $3 \mathrm{i}$ & $2-\mathrm{OH}$ & $4^{\prime}-\mathrm{OH}$ & 100 & $29.9 \pm 1.1$ & $36.3 \pm 3.9$ & $70.8 \pm 1.3$ \\
\hline $3 j$ & $4-\mathrm{OCH}_{3}$ & $4^{\prime}-\mathrm{OH}$ & 100 & $68.3 \pm 1.4$ & $44.1 \pm 3.6$ & $67.2 \pm 3.2$ \\
\hline $3 k$ & $4-\mathrm{CH}_{3}$ & $4^{\prime}-\mathrm{OH}$ & 100 & $65.4 \pm 3.4$ & $45.8 \pm 3.3$ & $59.03 \pm 6.2$ \\
\hline 31 & 3,4-dimethoxy & $4^{\prime}-\mathrm{OH}$ & $93.7 \pm 5.6$ & $65.3 \pm 2.0$ & $53.5 \pm 2.7$ & $67.8 \pm 3.5$ \\
\hline $3 \mathrm{~m}$ & 3,4,5-trimethoxy & $4^{\prime}-\mathrm{OH}$ & $88.0 \pm 3.1$ & $65.2 \pm 3.4$ & $84.6 \pm 1.1$ & $93.4 \pm 0.9$ \\
\hline $3 \mathrm{n}$ & $3-\mathrm{OH}$ & $4^{\prime}-\mathrm{OH}$ & 100 & $62.3 \pm 7.1$ & $33.4 \pm 3.5$ & $80.5 \pm 1.4$ \\
\hline 30 & $2-\mathrm{OCH}_{3}$ & $4^{\prime}-\mathrm{OH}$ & $92.4 \pm 2.1$ & $130.7 \pm 5.1$ & $43.8 \pm 1.6$ & $74.9 \pm 3.4$ \\
\hline $3 p$ & $2-\mathrm{OH}$ & $2^{\prime}-\mathrm{OH}$ & $95.1 \pm 3.0$ & $65.5 \pm 4.3$ & $41.1 \pm 3.7$ & $70.2 \pm 2.5$ \\
\hline $3 q$ & $3-\mathrm{OH}$ & $2^{\prime}-\mathrm{OH}$ & 100 & $30.7 \pm 1.2$ & $38.4 \pm 3.9$ & $36.1 \pm 3.5$ \\
\hline \multirow{3}{*}{$\begin{array}{c}\text { 3r } \\
\text { Indomethacin } \\
\text { Acetyl salicylic } \\
\text { acid (ASA) }\end{array}$} & $4-\mathrm{CH}_{3}$ & $2^{\prime}-\mathrm{OH}$ & 100 & $19.8 \pm 1.1$ & $39.8 \pm 3.2$ & $63.5 \pm 1.3$ \\
\hline & & & & $1.67 \pm 0.67$ & $42.0 \pm 1.1$ & \\
\hline & & & & $0.24 \pm 0.05$ & $21.0 \pm 0.6$ & \\
\hline
\end{tabular}

a Inhibition of platelet aggregation was assessed at $1 \mathrm{mM}$ concentration.

\subsection{Structure Activity Relationship}

The data reported in Table 1 show that all the compounds (3a-3r) at the concentration of $1 \mathrm{mM}$ inhibited platelet aggregation induced by ADP, AA, and collagen. The inhibition range for ADP and collagen were $30 \%-84.6 \%$ and $15.9 \%-93.4 \%$, respectively. When AA was used as a platelet aggregation inducer, the inhibition was increased and ranged from $74.7 \%$ to $100 \%$.

Compound $3 \mathrm{~m}$ with three-methoxy group on ring A inhibited platelet aggregation induced by all the three platelet inducers above $85 \%$. 
Since all compounds at concentration of $1 \mathrm{mM}$ were able to completely inhibit platelet aggregation induced by $\mathrm{AA}$, the $\mathrm{IC}_{50}$ values for these compounds were calculated (Table 1 ).

As shown in Table 1, all the compounds with hydroxyl substituent on the B ring show high activity $\left(\mathrm{IC}_{50}<69.1 \mu \mathrm{M}\right)$ except 3o. The results demonstrated that all the compounds with Schiff base and phenolic hydroxyl groups at the ortho position of ring $\mathrm{A}$ or $\mathrm{B}$ show $\mathrm{IC}_{50}$ values ranging between $19.8 \mu \mathrm{M}$ and $30.7 \mu \mathrm{M}$ except for $3 \mathbf{c}$ and $\mathbf{3 p}$. Compounds $\mathbf{3 i}$ and $\mathbf{3 q}$ exhibited satisfactory activity with $\mathrm{IC}_{50}$ values of $29.9 \mu \mathrm{M}$ and $30.7 \mu \mathrm{M}$, respectively. Compound $3 \mathbf{r}$ with $\mathrm{IC}_{50}$ value of $19.8 \mu \mathrm{M}$ was the most active compound.

\section{Materials and Methods}

\subsection{General Procedure for the Preparation of $\mathbf{3 a}-\mathbf{3 r}$}

The mixture of aromatic amine $(1 \mathrm{mmol})$ and aldehyde $(1 \mathrm{mmol})$ in water was stirred at room temperature. After completion of the reaction indicated by TLC (thin-layer chromatography), the obtained precipitate was filtered off and washed with water. The obtained precipitate was recrystallized from the appropriate solvent.

(E)-1-(4-Methoxyphenyl)-N-phenylmethanimine (3a). Yield 95\%; m.p. $48-50{ }^{\circ} \mathrm{C}$ (m.p. $49-50{ }^{\circ} \mathrm{C}$ [31]). ESI-MS m/z: $212[\mathrm{M}+\mathrm{H}]^{+}$. Anal. Calcd for $\mathrm{C}_{14} \mathrm{H}_{13} \mathrm{NO}: \mathrm{C} 79.59, \mathrm{H}$ 6.20, $\mathrm{N}$ 6.63, found C 79.57, $\mathrm{H}$ 6.21, N 6.64 .

(E)-2-Methoxy-4-[(phenylimino)methyl]phenol (3b). Yield 89\%. m.p. 159-162 ${ }^{\circ} \mathrm{C}$ (m.p. $\left.158-160{ }^{\circ} \mathrm{C}[32]\right)$. ESI-MS m/z: $228[\mathrm{M}+\mathrm{H}]^{+}$; Anal. Calcd for $\mathrm{C}_{14} \mathrm{H}_{13} \mathrm{NO}_{2}$ : C 73.99, $\mathrm{H}$ 5.77, $\mathrm{N}$ 6.16, found C 73.97, H 5.76, N 6.15 .

(E)-2-\{[(4-Methoxyphenyl)imino]methyl $\}$ phenol (3c). Yield 94\%. m.p. $76-77{ }^{\circ} \mathrm{C} ;{ }^{1} \mathrm{H}-\mathrm{NMR}(400 \mathrm{MHz}$, DMSO- $\left.d_{6}\right) \delta 13.32(\mathrm{~s}, 1 \mathrm{H}, \mathrm{OH}), 8.94(\mathrm{~s}, 1 \mathrm{H}, \mathrm{HC}=\mathrm{N}), 7.61(\mathrm{~m}, 1 \mathrm{H}, \mathrm{Ar}-\mathrm{H}), 7.42(\mathrm{~m}, 3 \mathrm{H}, \mathrm{Ar}-\mathrm{H}), 6.96$ $(\mathrm{m}, 4 \mathrm{H}, \mathrm{Ar}-\mathrm{H}), 3.79$ (s, 3H, $\left.\mathrm{OCH}_{3}\right) .{ }^{13} \mathrm{C}-\mathrm{NMR}\left(100 \mathrm{MHz}, \mathrm{DMSO}-d_{6}\right) \delta 161.72,160.62,158.99,141.19$, 133.25, 132.80, 123.09, 119.85, 119.53, 116.97, 115.13, 55.86; ESI-MS m/z: $228[\mathrm{M}+\mathrm{H}]^{+}$. Anal. Calcd for $\mathrm{C}_{14} \mathrm{H}_{13} \mathrm{NO}_{2}$ : C 73.99, H 5.77, N 6.16, found C 73.95, H 5.76, N 6.17.

(E)-N,1-bis(4-Methoxyphenyl)methanimine (3d). Yield 89\%. m.p. $154-155^{\circ} \mathrm{C}$ (m.p. $154{ }^{\circ} \mathrm{C}$ [33]). ESI-MS $m / z: 242[\mathrm{M}+\mathrm{H}]^{+}$. Anal. Calcd for $\mathrm{C}_{15} \mathrm{H}_{15} \mathrm{NO}_{2}: \mathrm{C}$ 74.67, H 6.27, N 5.81, found C 74.68, H 6.28, N 5.79.

(E)-1-(3,4-Dimethoxyphenyl)-N-(4-methoxyphenyl)methanimine (3e). Yield 85\%. m.p. 126-128 ${ }^{\circ} \mathrm{C} .{ }^{1} \mathrm{H}-\mathrm{NMR}$ $\left(400 \mathrm{MHz}, \mathrm{DMSO}-d_{6}\right) \delta 8.52(\mathrm{~s}, 1 \mathrm{H}, \mathrm{HC}=\mathrm{N}), 6.96-7.54(\mathrm{~m}, 7 \mathrm{H}, \mathrm{Ar}-\mathrm{H}), 3.77-3.83\left(\mathrm{~m}, 9 \mathrm{H}, \mathrm{OCH}_{3}\right) ;{ }^{13} \mathrm{C}-\mathrm{NMR}$ $\left(100 \mathrm{MHz}, \mathrm{DMSO}-d_{6}\right) \delta 158.46,158.05,151.97,149.45,144.89,129.77,124.08,122.66,115.46,114.95$, $114.85,111.74,109.68,56.07,55.88,55.73$. ESI-MS $m / z: 272[\mathrm{M}+\mathrm{H}]^{+}$. Anal. Calcd for $\mathrm{C}_{16} \mathrm{H}_{17} \mathrm{NO}_{3}$ : C 70.83, H 6.32, N 5.16, found C 70.81, H 6.31, N 5.17.

(E)-N-(4-Methoxyphenyl)-1-(p-tolyl)methanimine (3f). Yield 96\%. m.p. 87-88 ${ }^{\circ} \mathrm{C} .{ }^{1} \mathrm{H}-\mathrm{NMR}(400 \mathrm{MHz}$, DMSO- $\left.d_{6}\right) \delta 8.59(\mathrm{~s}, 1 \mathrm{H}, \mathrm{HC}=\mathrm{N}), 7.70-7.81(\mathrm{~m}, 2 \mathrm{H}, \mathrm{Ar}-\mathrm{H}), 7.31-7.42(\mathrm{~m}, 4 \mathrm{H}, \mathrm{Ar}-\mathrm{H}), 6.98(\mathrm{~m}, 2 \mathrm{H}, \mathrm{Ar}-\mathrm{H})$, $3.78\left(\mathrm{~s}, 3 \mathrm{H}, \mathrm{OCH}_{3}\right), 2.38\left(\mathrm{~s}, 3 \mathrm{H}, \mathrm{OCH}_{3}\right) .{ }^{13} \mathrm{C}-\mathrm{NMR}\left(100 \mathrm{MHz}, \mathrm{DMSO}-d_{6}\right) \delta 158.2,157.8,144.2,141.0$, $133.8,129.85,128.88,122.79,114.87,55.76,21.1$. ESI-MS $m / z: 226[\mathrm{M}+\mathrm{H}]^{+}$. Anal. Calcd for $\mathrm{C}_{15} \mathrm{H}_{15} \mathrm{NO}$ : C 79.97, H 6.71, N 6.22, found C 79.95, H 6.70, N 6.23.

(E)-2-Methoxy-4-\{[(4-methoxyphenyl)imino]methyl\}phenol (3g). Yield 94\%. m.p. $154-155{ }^{\circ} \mathrm{C}$ (m.p. $154{ }^{\circ} \mathrm{C}$ [34]). ESI-MS $m / z: 258[\mathrm{M}+\mathrm{H}]^{+}$. Anal. Calcd for $\mathrm{C}_{15} \mathrm{H}_{15} \mathrm{NO}_{3}: \mathrm{C} 70.02, \mathrm{H}$ 5.88, N 5.44, found C 70.01, H 5.89, N 5.43 .

(E)-4-[(3-Methoxybenzylidene)amino]phenol (3h). Yield $94 \%$. m.p. $165-167^{\circ} \mathrm{C}$ (m.p. $167^{\circ} \mathrm{C}$ [35]). ESI-MS $m / z: 228[\mathrm{M}+\mathrm{H}]^{+}$. Anal. Calcd for $\mathrm{C}_{14} \mathrm{H}_{13} \mathrm{NO}_{2}$ : C 73.99, H 5.77, N 6.16, found C 73.97, H 5.76, N 6.15.

(E)-2-\{[(4-Hydroxyphenyl)imino]methyl\}phenol (3i). Yield 98\%. m.p. $140-143{ }^{\circ} \mathrm{C} .{ }^{1} \mathrm{H}-\mathrm{NMR}(400 \mathrm{MHz}$, DMSO- $\left.d_{6}\right) \delta 13.44(\mathrm{~s}, 1 \mathrm{H}, \mathrm{OH}), 9.72(\mathrm{~s}, 1 \mathrm{H}, \mathrm{OH}), 8.94(\mathrm{~s}, 1 \mathrm{H}, \mathrm{HC}=\mathrm{N}), 7.58(\mathrm{~m}, 1 \mathrm{H}, \mathrm{Ar}-\mathrm{H}), 7.31-7.37(\mathrm{~m}$, 
3H, Ar-H), 6.84-6.95 (m, 4H, Ar-H). ${ }^{13} \mathrm{C}-\mathrm{NMR}\left(100 \mathrm{MHz}, \mathrm{DMSO}-d_{6}\right) \delta 160.67,160.60,157.41,139.65$, 133.00, 132.67, 123.12, 119.89, 119.46, 116.92, 116.43, 116.01, 115.85. ESI-MS m/z: $214[\mathrm{M}+\mathrm{H}]^{+}$. Anal. Calcd for $\mathrm{C}_{13} \mathrm{H}_{11} \mathrm{NO}_{2}$ : C 73.23, H 5.20, N 6.57, found C 73.21, H 5.21, N 6.56.

(E)-4-[(4-Methoxybenzylidene)amino]phenol (3j). Yield 95\%. m.p. $187-188^{\circ} \mathrm{C}$ (m.p. $189^{\circ} \mathrm{C}$ [36]). ESI-MS $m / z: 228[\mathrm{M}+\mathrm{H}]^{+}$. Anal. Calcd for $\mathrm{C}_{14} \mathrm{H}_{13} \mathrm{NO}_{2}$ : C 73.99, H 5.77, N 6.16, found C 74.20, H 5.77, N 6.15.

(E)-4-[(4-Methylbenzylidene)amino]phenol (3k). Yield 94\%. m.p. $152-153{ }^{\circ} \mathrm{C} ;{ }^{1} \mathrm{H}-\mathrm{NMR}(400 \mathrm{MHz}$, DMSO- $\left.d_{6}\right) \delta 9.51(\mathrm{~s}, 1 \mathrm{H}, \mathrm{OH}), 8.55(\mathrm{~s}, 1 \mathrm{H}, \mathrm{HC}=\mathrm{N}), 7.78(\mathrm{~m}, 2 \mathrm{H}, \mathrm{Ar}-\mathrm{H}), 7.18-7.30(\mathrm{~m}, 4 \mathrm{H}, \mathrm{Ar}-\mathrm{H}), 6.80(\mathrm{~m}$, 2H, Ar-H), $2.36\left(\mathrm{~s}, 3 \mathrm{H}, \mathrm{CH}_{3}\right) ;{ }^{13} \mathrm{C}-\mathrm{NMR}\left(100 \mathrm{MHz}, \mathrm{DMSO}-d_{6}\right) \delta 157.51,156.57,143.19,141.24,134.36$, 129.82, 128.73, 122.86, 116.15, 21.59; ESI-MS m/z: $212[\mathrm{M}+\mathrm{H}]^{+}$. Anal. Calcd for $\mathrm{C}_{14} \mathrm{H}_{13} \mathrm{NO}$ : C 79.59, H 6.20, N 6.63, found C 79.45, H 6.19, N 6.62.

(E)-4-[(3,4-Dimethoxybenzylidene)amino]phenol (31). Yield 92\%. m.p. $155-156{ }^{\circ} \mathrm{C}$ (m.p. $155{ }^{\circ} \mathrm{C}$ [37]). ESI-MS $m / z: 258[\mathrm{M}+\mathrm{H}]^{+}$. Anal. Calcd for $\mathrm{C}_{15} \mathrm{H}_{15} \mathrm{NO}_{3}$ : C 70.02, H 5.88, N 5.44, found C 70.10, H 5.87, N 5.45 .

(E)-4-[(3,4,5-Trimethoxybenzylidene)amino]phenol (3m). Yield 96\%. m.p. $140-142{ }^{\circ} \mathrm{C} .{ }^{1} \mathrm{H}-\mathrm{NMR}(400 \mathrm{MHz}$, DMSO- $\left._{6}\right) \delta 9.53(\mathrm{~s}, 1 \mathrm{H}, \mathrm{OH}), 8.51(\mathrm{~s}, 1 \mathrm{H}, \mathrm{HC}=\mathrm{N}), 7.18-7.23(\mathrm{~m}, 4 \mathrm{H}, \mathrm{Ar}-\mathrm{H}), 6.81(\mathrm{~m}, 2 \mathrm{H}, \mathrm{Ar}-\mathrm{H}), 3.85(\mathrm{~s}$, $\left.6 \mathrm{H}, \mathrm{OCH}_{3}\right), 3.73\left(\mathrm{~s}, 3 \mathrm{H}, \mathrm{OCH}_{3}\right) .{ }^{13} \mathrm{C}-\mathrm{NMR}\left(100 \mathrm{MHz}, \mathrm{DMSO}-d_{6}\right) \delta 157.42,156.60,153.58,143.06,140.31$, $132.44,122.85,116.19,115.87,107.19,105.88,60.61,56.50,56.36$; ESI-MS $m / z: 288[\mathrm{M}+\mathrm{H}]^{+}$. Anal. Calcd for $\mathrm{C}_{16} \mathrm{H}_{17} \mathrm{NO}_{4}$ : C 66.89, H 5.96, N 4.88, found C 66.84, H 5.95, N 4.88.

(E)-3-\{[(4-Hydroxyphenyl)imino]methyl\}phenol (3n). Yield 94\%. m.p. 191-193 ${ }^{\circ} \mathrm{C} .{ }^{1} \mathrm{H}-\mathrm{NMR}(400 \mathrm{MHz}$, DMSO-d $\left.d_{6}\right) \delta 9.69(\mathrm{~s}, 1 \mathrm{H}, \mathrm{OH}), 9.55(\mathrm{~s}, 1 \mathrm{H}, \mathrm{OH}), 8.51(\mathrm{~s}, 1 \mathrm{H}, \mathrm{HC}=\mathrm{N}), 7.19-7.37(\mathrm{~m}, 5 \mathrm{H}, \mathrm{Ar}-\mathrm{H}), 6.83-6.91$ $(\mathrm{m}, 3 \mathrm{H}, \mathrm{Ar}-\mathrm{H}) .{ }^{13} \mathrm{C}-\mathrm{NMR}\left(100 \mathrm{MHz}\right.$, DMSO- $\left.d_{6}\right) \delta 158.10,157.66,156.70,143.04,138.29,130.23,122.95$, 120.44, 118.64, 116.19, 115.90, 114.35; ESI-MS m/z: $214[\mathrm{M}+\mathrm{H}]^{+}$. Anal. Calcd for $\mathrm{C}_{13} \mathrm{H}_{11} \mathrm{NO}_{2}: \mathrm{C}$ 73.23, H 5.20, N 6.57, found C 73.21, H 5.21, N 6.58 .

(E)-4-[(2-Methoxybenzylidene)amino]phenol (3o). Yield 92\%. m.p. $168-169^{\circ} \mathrm{C}$ (m.p. $168^{\circ} \mathrm{C}$ [35]). ESI-MS $m / z: 228[\mathrm{M}+\mathrm{H}]^{+}$. Anal. Calcd for $\mathrm{C}_{14} \mathrm{H}_{13} \mathrm{NO}_{2}$ : C 73.99, H 5.77, N 6.16, found C 73.94, H 5.78, N 6.15.

(E)-2-[(2-Hydroxybenzylidene)amino]phenol (3p). Yield 98\%. m.p. $141-143{ }^{\circ} \mathrm{C} .{ }^{1} \mathrm{H}-\mathrm{NMR}(400 \mathrm{MHz}$, DMSO-d $\left.d_{6}\right) \delta 13.81(\mathrm{~s}, 1 \mathrm{H}, \mathrm{OH}), 9.77(\mathrm{~s}, 1 \mathrm{H}, \mathrm{OH}), 8.96(\mathrm{~s}, 1 \mathrm{H}, \mathrm{HC}=\mathrm{N}), 7.13-7.61(\mathrm{~m}, 4 \mathrm{H}, \mathrm{Ar}-\mathrm{H}), 6.89-6.95$ $(\mathrm{m}, 4 \mathrm{H}, \mathrm{Ar}-\mathrm{H}) .{ }^{13} \mathrm{C}-\mathrm{NMR}\left(100 \mathrm{MHz}, \mathrm{DMSO}-d_{6}\right) \delta 162.13,161.20,151.56,135.40,133.31,132.78,128.53$, $120.09,120.04,119.96,119.21,117.16,116.98$. ESI-MS $m / z: 214[\mathrm{M}+\mathrm{H}]^{+}$. Anal. Calcd for $\mathrm{C}_{13} \mathrm{H}_{11} \mathrm{NO}_{2}$ : C 73.23, H 5.20, N 6.57, found C 73.24, H 5.19, N 6.56.

(E)-2-[(3-Hydroxybenzylidene)amino]phenol (3q). Yield 99\%. m.p. $122-124{ }^{\circ} \mathrm{C}$ (m.p. $\left.122.5-123.5^{\circ} \mathrm{C}[38]\right)$. ESI-MS $m / z: 214[\mathrm{M}+\mathrm{H}]^{+}$. Anal. Calcd for $\mathrm{C}_{13} \mathrm{H}_{11} \mathrm{NO}_{2}: \mathrm{C} 73.23, \mathrm{H} 5.20, \mathrm{~N}$ 6.57, found C 73.24, H 5.19, N 6.55 .

(E)-2-[(4-Methylbenzylidene)amino]phenol (3r). Yield 98\%. m.p. 107-108 ${ }^{\circ} \mathrm{C}$ (m.p. $108.5^{\circ} \mathrm{C}$ [39]). ESI-MS $m / z: 212[\mathrm{M}+\mathrm{H}]^{+}$. Anal. Calcd for $\mathrm{C}_{14} \mathrm{H}_{13} \mathrm{NO}$ : C 79.59, H 6.20, N 6.63, found C 79.58, H 6.19, N 6.64.

\subsection{Anti-Platelet Assay}

The anti-platelet aggregation activity of the synthesized compounds was evaluated on an APACT 4004 aggregometer (LABiTec, Ahrensburg, Germany), according to the method described before $[29,40,41]$. Compounds (3a-3r) were added to platelet-rich plasma (PRP) and were incubated for $5 \mathrm{~min}$ at $37^{\circ} \mathrm{C}$. Adenosine diphosphate (ADP), arachidonic acid, and collagen were added separately as platelet aggregation inducers at a final concentration of $5 \mu \mathrm{M}, 1.35 \mu \mathrm{M}$, and $2.5 \mu \mathrm{g} \cdot \mathrm{mL}^{-1}$, respectively. The aggregation procedure was monitored for $5 \mathrm{~min}$. Compounds were screened at a concentration of $1 \mathrm{mM}$ in DMSO. The $\mathrm{IC}_{50}$ values against AA were determined for the synthesized compounds. Each experiment was carried out in triplicate and the results are shown as a mean \pm standard error of mean (SEM). 


\section{Conclusions}

In the present study, a series of resveratrol derivatives was synthesized and their antiplatelet activity was evaluated against ADP, AA, and collagen as platelet aggregation inducers. Compound $3 \mathrm{r}$ was the most active agent against AA and, therefore, possesses the potential to be considered a lead compound for future studies and further investigations. Lastly, the imine functional group may be qualified as an effective replacement for the double bond in resveratrol for anti-platelet aggregation pharmacophore.

Supplementary Materials: The following are available online http://www.mdpi.com/1422-8599/2019/1/ M1039/s1, Figure S1: Qualitative data analysis report.

Author Contributions: M.B., M.S., M.E., and F.K. carried out the experiments, analyzed the results, and wrote the manuscript. S.V., M.I., and J.S.-R. contributed to the discussion of results and critically reviewed the manuscript. All the authors read and approved the final manuscript.

Funding: This research received no external funding.

Conflicts of Interest: The authors declare no conflict of interest.

\section{References}

1. Organization, W.H. Cardiovascular Diseases. Fact Sheet 317. 2007. Available online: https:/ /www.who.int/ news-room/fact-sheets/detail/cardiovascular-diseases-(cvds) (accessed on 17 May 2017).

2. Palmer, M.; Sutherland, J.; Barnard, S.; Wynne, A.; Rezel, E.; Doel, A.; Grigsby-Duffy, L.; Edwards, S.; Russell, S.; Hotopf, E. The effectiveness of smoking cessation, physical activity/diet and alcohol reduction interventions delivered by mobile phones for the prevention of non-communicable diseases: A systematic review of randomised controlled trials. PLoS ONE 2018, 13, e0189801. [CrossRef] [PubMed]

3. Ren, Y.; Patel, K.; Crane, T. A review of antiplatelet drugs, coronary artery diseases and cardiopulmonary bypass. J. Extr.-Corporeal Technol. 2010, 42, 103.

4. Guthrie, R. Review and management of side effects associated with antiplatelet therapy for prevention of recurrent cerebrovascular events. Adv. Ther. 2011, 28, 473. [CrossRef] [PubMed]

5. Sharifi-Rad, M.; Nazaruk, J.; Polito, L.; Morais-Braga, M.F.B.; Rocha, J.E.; Coutinho, H.D.M.; Salehi, B.; Tabanelli, G.; Montanari, C.; del Mar Contreras, M. Matricaria genus as a source of antimicrobial agents: From farm to pharmacy and food applications. Microbiol. Res. 2018. [CrossRef] [PubMed]

6. Sharifi-Rad, M.; Fokou, P.; Sharopov, F.; Martorell, M.; Ademiluyi, A.; Rajkovic, J.; Salehi, B.; Martins, N.; Iriti, M.; Sharifi-Rad, J. Antiulcer agents: From plant extracts to phytochemicals in healing promotion. Molecules 2018, 23, 1751. [CrossRef] [PubMed]

7. Salehi, B.; Albayrak, S.; Antolak, H.; Kręgiel, D.; Pawlikowska, E.; Sharifi-Rad, M.; Uprety, Y.; Tsouh Fokou, P.; Yousef, Z.; Amiruddin Zakaria, Z. Aloe genus plants: From farm to food applications and phytopharmacotherapy. Int. J. Mol. Sci. 2018, 19, 2843. [CrossRef] [PubMed]

8. Prakash, M.A.; Sharifi-Rad, M.; Shariati, M.; Mabkhot, Y.; Al-Showiman, S.; Rauf, A.; Salehi, B.; Župunski, M.; Sharifi-Rad, M.; Gusain, P. Bioactive compounds and health benefits of edible Rumex species-a review. Cell. Mol. Biol. (Noisy-le-Grand, France) 2018, 64, 27. [CrossRef]

9. Mishra, A.; Saklani, S.; Salehi, B.; Parcha, V.; Sharifi-Rad, M.; Milella, L.; Iriti, M.; Sharifi-Rad, J.; Srivastava, M. Satyrium nepalense, a high altitude medicinal orchid of indian himalayan region: Chemical profile and biological activities of tuber extracts. Cell. Mol. Biol. (Noisy-le-Grand, France) 2018, 64, 35-43. [CrossRef]

10. Guo, Z. The modification of natural products for medical use. Acta Pharm. Sinica B 2017, 7, 119-136. [CrossRef]

11. Bovicelli, P.; Bernini, R.; Antonioletti, R.; Mincione, E. Selective halogenation of flavanones. Tetrahedron Lett. 2002, 43, 5563-5567. [CrossRef]

12. Salehi, B.; Valussi, M.; Jugran, A.K.; Martorell, M.; Ramírez-Alarcón, K.; Stojanović-Radić, Z.Z.; Antolak, H.; Kręgiel, D.; Mileski, K.S.; Sharifi-Rad, M. Nepeta species: From farm to food applications and phytotherapy. Trends Food Sci. Technol. 2018. [CrossRef]

13. Olas, B.; Wachowicz, B. Resveratrol, a phenolic antioxidant with effects on blood platelet functions. Platelets 2005, 16, 251-260. [CrossRef] [PubMed] 
14. Li, S.-Y.; Wang, X.-B.; Kong, L.-Y. Design, synthesis and biological evaluation of imine resveratrol derivatives as multi-targeted agents against Alzheimer's disease. Eur. J. Med. Chem. 2014, 71, 36-45. [CrossRef] [PubMed]

15. Iriti, M.; Vitalini, S. Chemical diversity of grape products, a complex blend of bioactive secondary metabolites. Nat. Prod. J. 2011, 1, 71-74.

16. Shen, M.Y.; Hsiao, G.; Liu, C.L.; Fong, T.H.; Lin, K.H.; Chou, D.S.; Sheu, J.R. Inhibitory mechanisms of resveratrol in platelet activation: Pivotal roles of p38 mapk and no/cyclic gmp. Br. J. Haematology 2007, 139, 475-485. [CrossRef] [PubMed]

17. Varoni, E.M.; Lo Faro, A.F.; Sharifi-Rad, J.; Iriti, M. Anticancer molecular mechanisms of resveratrol. Front. Nutr. 2016, 3, 8. [CrossRef] [PubMed]

18. Orsini, F.; Pelizzoni, F.; Verotta, L.; Aburjai, T.; Rogers, C.B. Isolation, synthesis, and antiplatelet aggregation activity of resveratrol 3-O- $\beta$-D-glucopyranoside and related compounds. J. Nat. Prod. 1997, 60, 1082-1087. [CrossRef]

19. Dutra, L.A.; Guanaes, J.F.O.; Johmann, N.; Pires, M.E.L.; Chin, C.M.; Marcondes, S.; Dos Santos, J.L. Synthesis, antiplatelet and antithrombotic activities of resveratrol derivatives with no-donor properties. Bioorg. Med. Chem. Lett. 2017, 27, 2450-2453. [CrossRef]

20. Tehrani, K.H.M.E.; Sardari, S.; Mashayekhi, V.; Zadeh, M.E.; Azerang, P.; Kobarfard, F. One pot synthesis and biological activity evaluation of novel schiff bases derived from 2-hydrazinyl-1,3,4-thiadiazole. Chem. Pharm. Bull. 2013, 61, 160-166. [CrossRef]

21. Akhlaghi, M.F.; Amidi, S.; Esfahanizadeh, M.; Daeihamed, M.; Kobarfard, F. Synthesis of n-arylmethyl substituted indole derivatives as new antiplatelet aggregation agents. Iranian J. Pharm. Res. 2014, 13, 35.

22. Lourenço, A.L.; Salvador, R.R.; Silva, L.A.; Saito, M.S.; Mello, J.F.; Cabral, L.M.; Rodrigues, C.R.; Vera, M.A.; Muri, E.M.; de Souza, A.M. Synthesis and mechanistic evaluation of novel $N^{\prime}$-benzylidene-carbohydrazide- $1 H$-pyrazolo $[3,4-b]$ pyridine derivatives as non-anionic antiplatelet agents. Eur. J. Med. Chem. 2017, 135, 213-229. [CrossRef] [PubMed]

23. Mirfazli, S.S.; Kobarfard, F.; Firoozpour, L.; Asadipour, A.; Esfahanizadeh, M.; Tabib, K.; Shafiee, A.; Foroumadi, A. N-substituted indole carbohydrazide derivatives: Synthesis and evaluation of their antiplatelet aggregation activity. DARU J. Pharm. Sci. 2014, 22, 65. [CrossRef] [PubMed]

24. Mirfazli, S.S.; Khoshneviszadeh, M.; Jeiroudi, M.; Foroumadi, A.; Kobarfard, F.; Shafiee, A. Design, synthesis and qsar study of arylidene indoles as anti-platelet aggregation inhibitors. Med. Chem. Res. 2016, 25, 1-18. [CrossRef]

25. Tehrani, K.H.M.E.; Zadeh, M.E.; Mashayekhi, V.; Hashemi, M.; Kobarfard, F.; Gharebaghi, F.; Mohebbi, S. Synthesis, antiplatelet activity and cytotoxicity assessment of indole-based hydrazone derivatives. Iranian J. Pharm. Res. 2015, 14, 1077.

26. Kalhor, N.; Mardani, M.; Abdollahzadeh, S.; Vakof, M.; Zadeh, M.E.; Tehrani, K.H.M.E.; Kobarfard, F.; Mohebbi, S. Novel N-substituted ((1H-indol-3-yl)methylene)benzohydrazides and ((1H-indol-3-yl)methylene)-2-phenylhydrazines: Synthesis and antiplatelet aggregation activity. Bull. Korean Chem. Soc. 2015, 36, 2632-2639. [CrossRef]

27. Mashayekhi, V.; Tehrani, K.H.M.E.; Amidi, S.; Kobarfard, F. Synthesis of novel indole hydrazone derivatives and evaluation of their antiplatelet aggregation activity. Chem. Pharm. Bull. 2013, 61, 144-150. [CrossRef] [PubMed]

28. Lima, L.M.; Barreiro, E.J. Bioisosterism: A useful strategy for molecular modification and drug design. Curr. Med. Chem. 2005, 12, 23-49. [CrossRef] [PubMed]

29. Amidi, S.; Kobarfard, F.; Moghaddam, A.B.; Tabib, K.; Soleymani, Z. Electrochemical synthesis of novel 1, 3-indandione derivatives and evaluation of their antiplatelet aggregation activities. Iranian J. Pharm. Res. 2013, 12, 91.

30. Born, G.V.R. Aggregation of blood platelets by adenosine diphosphate and its reversal. Nature 1962, 194, 927-929. [CrossRef]

31. Iovel, I.; Golomba, L.; Fleisher, M.; Popelis, J.; Grinberga, S.; Lukevics, E. Hydrosilylation of (hetero) aromatic aldimines in the presence of a Pd (I) complex. Chem. Heterocycl. Compd. 2004, 40, 701-714. [CrossRef]

32. Cheng, L.-X.; Tang, J.-J.; Luo, H.; Jin, X.-L.; Dai, F.; Yang, J.; Qian, Y.-P.; Li, X.-Z.; Zhou, B. Antioxidant and antiproliferative activities of hydroxyl-substituted schiff bases. Bioorg. Med. Chem. Lett. 2010, 20, 2417-2420. [CrossRef] [PubMed] 
33. Nassar, A.; Hassan, A.; Ibraheem, N.; Hekal, B. Synthesis and comparative studies of cyclopalladated complexes with ortho c-h activation of aromatic rings bearing electron donating and electron withdrawing groups. Synth. React. Inorg. Met.-Org. Nano-Metal Chem. 2015, 45, 813-820. [CrossRef]

34. Gebretekle, D.; Tadesse, A.; Upadhyay, R.; Dekebo, A. Synthesis, characterization and antimicrobial evaluation of some schiff bases and their thiazolidinone products. Oriental J. Chem. 2012, 28, 1791-1796. [CrossRef]

35. Grammaticakis, P.; Texier, H. Contribution al'étude de l'absorption dans l'ultraviolet moyen et le visible de derivés fonctionnels azotés de quelques aldéhydes et cétones aromatiques. X.-aniles (premier mémoire). Bull. Soc. Chim. Fr. 1971, 38, 1323-1330.

36. Oliveira Calil, N.; Senra Goncalves de Carvalho, G.; Farah da Silva, A.; David da Silva, A.; Rezende Barbosa Raposo, N. Antioxidant activity of synthetic resveratrol analogs: A structure-activity insight. Lett. Drug Des. Discovery 2012, 9, 676-679. [CrossRef]

37. Ceyhan, G.; Köse, M.; Tümer, M.; Demirtaş, İ. Anticancer, photoluminescence and electrochemical properties of structurally characterized two imine derivatives. Spectrochim. Acta Part A Mol. Biomolecular. Spectrosc. 2015, 149, 731-743. [CrossRef] [PubMed]

38. Kazantsev, A.V.; Aksartov, M.M.; Zakharkin, L.I. 1,4-Addition der lithium-und magnesiumderivate von o-carboranen an alpha, beta-nitroolefine. Zh. Obshch. Khim. [J. Gen. Chem. USSR (Engl. Transl.)] 1971, 41, 57.

39. Singleton, F.; Pollard, C. Reactions of aldehydes with amines. I. With o-aminophenol. J. Am. Chem. Soc. 1940, 62, 2288-2289. [CrossRef]

40. Lorigooini, Z.; Ayatollahi, S.A.; Amidi, S.; Kobarfard, F. Evaluation of anti-platelet aggregation effect of some Allium species. Iranian J. Pharm. Res. 2015, 14, 1225.

41. Amidi, S.; Esfahanizadeh, M.; Tabib, K.; Soleimani, Z.; Kobarfard, F. Rational design and synthesis of 1-(arylideneamino)-4-aryl-1H-imidazole-2-amine derivatives as antiplatelet agents. Chem. Med. Chem. 2017, 12, 962-971. [CrossRef]

(C) 2018 by the authors. Licensee MDPI, Basel, Switzerland. This article is an open access article distributed under the terms and conditions of the Creative Commons Attribution (CC BY) license (http:/ / creativecommons.org/licenses/by/4.0/). 